\title{
Quilotórax a Tensión: Reporte de Dos Casos
}

\author{
CONSTANZA PINOCHET V. ${ }^{1}$, ALEJANDRO DONOSO F. ${ }^{2}$, PABLO CRUCES R. ${ }^{3}$ \\ 1. Becada de Pediatría, Pontificia Universidad Católica de Chile. \\ 2. Médico. Área de Cuidados Críticos. Unidad de Gestión Clínica del Niño. Hospital Padre Hurtado. \\ 3. Médico. Programa de Medicina Intensiva Infantil. Universidad del Desarrollo Clínica Alemana de Santiago.
}

\section{ABSTRACT \\ Tension Chylothorax: Two Case Reports}

Thoracic duct injury is a well described entity as complication from cardiothoracic surgery. However tension chylothorax is rare, and may become a life-threating condition. Objective: To present 2 pediatric patients who developed hemodynamic and respiratory failures secondary to chylothorax. Patients: The first patient was a 2-month-old boy who developed chylothorax three weeks after a Norwood-Sano surgery; he showed a severe respiratory and hemodynamic collapse. The second patient was a one-month old baby who developed an acute respiratory failure and oliguria two days after a patent ductus arteriosus surgery. In both cases the chest tube placement resulted in the release of chyle under pressure and resolution of the symptoms. Conclusions: These two cases demostrate how chylothorax may provoke severe hemodynamic and respiratory effects. Early recognition and treatment of this condition is important for improved outcome.

(Key words: Chyle, chylothorax, tension chylothorax, pleural efusión).

Rev Chil Pediatr 2009; 80 (3): 256-260

\section{RESUMEN}

El daño del ducto toráxico que ocasiona un quilotórax es una complicación bien conocida y documentada de las cirugías cardiotoráxicas. Sin embargo, el desarrollo de quilotórax a tensión es raramente reportado, siendo un evento que puede poner en riesgo la vida del paciente. Objetivo: Comunicar 2 pacientes, quienes presentaron compromiso hemodinámico y respiratorio de carácter grave, secundario al desarrollo de quilotórax. Casos: El primer paciente, de dos meses de edad, ocurrió luego de tres semanas de efectuarse una cirugía de Norwood-Sano, quien presentó un grave colapso ventilatorio y hemodinámico. El segundo paciente, de 1 mes de edad, desarrolló una falla respiratoria aguda y oliguria dos días posterior al cierre de un ductus arterioso persistente. En ambos casos la instalación de un tubo pleural resultó en la liberación de quilo a gran presión y una rápida resolución de los síntomas. Conclusión: Los dos casos aquí reportados evidencian que el quilotórax puede presentar efectos hemodinámicos y respiratorios deletéreos. El pronto reconocimiento y tratamiento de esta entidad son esenciales para el óptimo pronóstico del paciente.

(Palabras clave: Quilo, quilotórax, quilotórax a tensión, derrame pleural).

Rev Chil Pediatr 2009; 80 (3): 256-260

Trabajo recibido el 10 de octubre de 2008, devuelto para corregir el 21 de enero de 2009, segunda versión el 09 de marzo de 2009, aceptado para publicación el 16 de abril de 2009.

Correspondencia a:

Dr. Alejandro Donoso F.

E-mail: adonoso@hurtadohosp.cl 


\section{Introducción}

En la población infantil la causa más común de ocupación del espacio pleural corresponde a los derrames paraneumónicos, sin embargo, existen diversas causas que pueden dar cuenta de un síndrome de ocupación pleural ${ }^{1}$.

Quilotórax es la acumulación de quilo (fluido linfático de origen intestinal) en el espacio pleural ocasionado por la ruptura o bloqueo del ducto toráxico o sus tributarios linfáticos. El quilotórax puede ser ocasionado por causa traumática o no traumática, y es una conocida complicación de cirugía toráxica o de un trauma toráxico significativo; siendo sus consecuencias más conocidas las nutricionales, metabólicas e inmunológicas.

La acumulación de gas que rápidamente se expande (neumotórax a tensión), es la causa más frecuente de ocupación pleural suficiente como para causar deterioro ventilatorio y hemodinámico. El incremento de las presiones dentro del espacio pleural causan compromiso hemodinámico ya sea este ocasionado por disminución del retorno venoso a las cavidades izquierdas o la directa compresión de la fosa pericárdica, o secundariamente a través de los efectos en la función ventilatoria originado por la hipoxemia y/o hipercarbia resultante. Todos estos efectos pueden ser más rápidos y graves en niños que en la población adulta, dado lo relativamente mas fácil de producirse en los primeros desplazamiento mediastínico ${ }^{2}$.

Existen diversas causas que pudiesen originar esta misma condición fisiopatológica subyacente, como ha sido comunicado para pioneumotórax $^{3,4}$, gastrotórax ${ }^{5}$, hidrotórax ${ }^{6}$ y urohemotórax ${ }^{7}$. El desarrollo de quilotórax a tensión luego de cirugía toráxica es una rara complicación, pero que debe de ser conocida.

El objetivo de la presente comunicación es reportar dos casos de pacientes ingresados al Área de Cuidados Críticos del Hospital Padre Hurtado, que desarrollaron colapso hemodinámico y/o ventilatorio en relación a quilotórax a tensión, situación que puso en riesgo la vida de estos pacientes.

\section{Caso clínico 1}

RN término de 10 días de vida, sexo masculino, con PN 2890 g, con diagnóstico prenatal de ventrículo único. A los 12 días de vida se efectuó Norwood-Sano (vide infra), sin inconvenientes. Requirió ventilación mecánica invasiva (VMI) los primeros 10 días del postoperatorio, alcanzándose a alimentar durante una semana. A los 18 días del postoperatorio se trasladó a nuestro hospital desde centro cardioquirúrgico en buenas condiciones generales (saturación hemoglobina $85 \%$ ) y con un peso de $3,1 \mathrm{~kg}$; en tratamiento farmacológico con digoxina, captoprilo y furosemida. A las 24 horas de ingresado, (día 19 postoperatorio), presentó brusca desaturación hasta $60 \%$, con frecuencia respiratoria de $60 / \mathrm{min}$, sin bradicardia; evolucionando con insuficiencia respiratoria aguda. Por lo anterior se traslado a UCI, donde ingresó con disminución de la perfusión periférica, cianosis generalizada, pulsos débiles y a la auscultación pulmonar destacaba franca disminución del murmullo pulmonar a derecha. Los gases en sangre arterial mostraron $\mathrm{pH}$ de $6,8, \mathrm{PaCO}_{2}$ de $135 \mathrm{mmHg}$, procediéndose a intubación y VM. La radiografía de tórax mostró imágenes de ocupación pleural a derecha sugerentes de derrame (figura 1). Se efectuó toracocentesis evacuadora dando salida a presión de $239 \mathrm{ml}$ de líquido de aspecto lechoso durante el primer día (figura 2). El análisis del líquido reveló 10000 leucocitos $/ \mathrm{mm}^{3}$ (95\% de linfocitos), $\mathrm{pH}$ de 7,5; triglicéridos de $660 \mathrm{mg} / \mathrm{dl}$; glucosa de $81 \mathrm{mg} / \mathrm{dl}$; $3,2 \mathrm{~g} / \mathrm{dl}$ de proteínas, lo que confirmó el diagnóstico de quilotórax. El paciente no presentó shock séptico durante la hospitalización por lo que se descartó este como factor contribuyente al desarrollo del quilotórax. La evolución clínica posterior fue satisfactoria, extubándose a los 3 días desde la instalación del drenaje y este se pudo retirar definitivamente a la semana de evolución. Se indicó dieta hipograsa, la que recibió por 3 semanas posterior al retiro del drenaje pleural, con lo que no se reprodujo el quilotórax. El paciente fue dado de alta en buenas condiciones, asintomático, al día 35 de hospitalización.

Norwood-Sano: ascenso de arteria aorta hipoplásica y conexión de esta al tronco de la arteria pulmonar + unión entre ventrículo derecho y arteria pulmonar (shunt de Sano), con lo que se pretende que el ventrículo derecho actúe como ventrículo sistémico. 


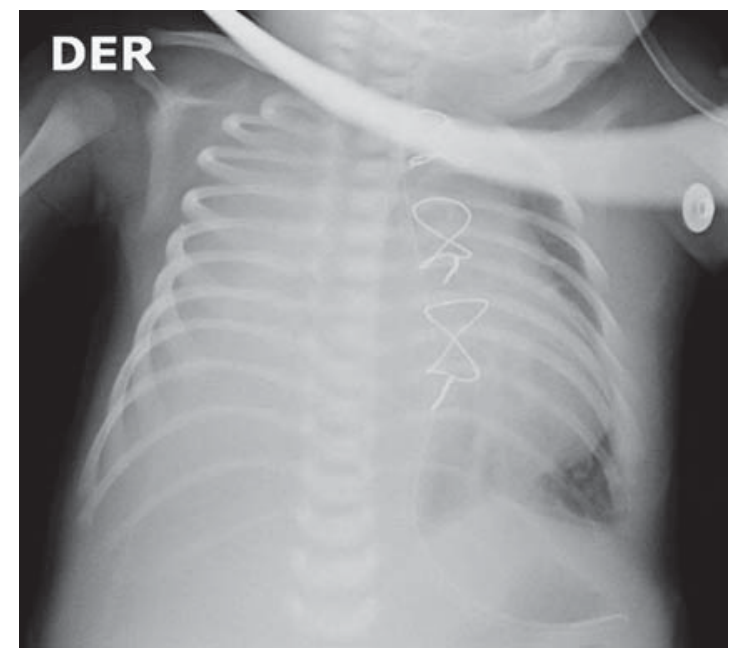

Figura 1. Radiografía de tórax (AP) que muestra completa opacidad del hemitórax derecho con desplazamiento de estructuras mediastínicas y corazón a izquierda, en paciente con quilotórax a tensión.

\section{Caso clínico 2}

Lactante de 2 meses de edad, sexo femenino, $3,1 \mathrm{~kg}$, con antecedente de prematurez (36 semanas), adecuado para la edad gestacional, PN 2,4 kg. Se diagnosticó ductus arteriosos persistente, el cual fue operado a las cinco semanas de vida. El postoperatorio inmediato cursó sin intercurrencias, lográndose extubación satisfactoria. A las 48 horas de evolución presentó taquicardia de 184 lat/min, PA de 87/51 $\mathrm{mmHg}$, oliguria y frecuencia respiratoria en 64 resp/min, saturación de $92 \%$ con $\mathrm{FiO}_{2}$ de $0,40 \%$, evolucionando con falla respiratoria aguda, por lo cual debió ser conectado a ventilación mecánica invasiva (VMI), y se optimizó la precarga con $30 \mathrm{ml} / \mathrm{kg}$ de fluidos. Radiografía de tórax evidenció opacidad de todo el campo pulmonar izquierdo. Se complementó estudio con ecografía, la que mostró un significativo derrame pleural izquierdo. Se instaló drenaje pleural (12 French), el cual dio salida a $100 \mathrm{ml}$ de líquido espeso a gran presión. El líquido obtenido resultó de aspecto turbio, color de tonalidad amarilla y con citoquímico con los siguientes resultados: 15000 leucocitos $/ \mathrm{mm}^{3}$ (90\% de linfocitos), $\mathrm{pH}$ 7,29; triglicéridos de $1103 \mathrm{mg} / \mathrm{dl}$; glucosa 70 $\mathrm{mg} / \mathrm{dl}$, proteínas $2,5 \mathrm{~g} / \mathrm{dl}$; albúmina $1,8 \mathrm{~g} / \mathrm{dl}$ y LDH de 342 U/1 lo que corroboró el diagnóstico

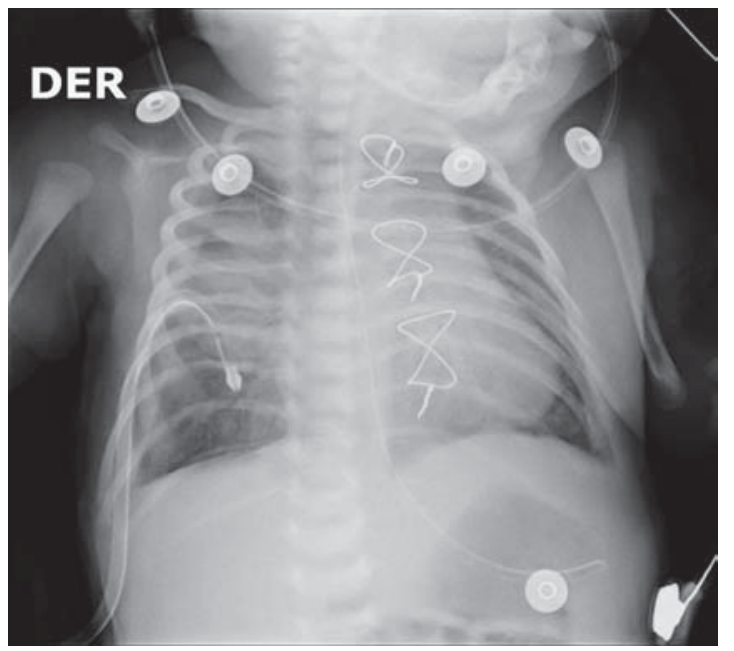

Figura 2. Radiografía de tórax (AP) que muestra reexpansión campo pulmonar derecho luego de instalación de tubo de drenaje pleural.

de quilotórax. Luego de la toracocentesis se logró reexpansión completa del pulmón izquierdo, con rápida disminución de los parámetros ventilatorios y observándose caída de frecuencia cardíaca y normalización de la presión arterial con una pronta mejoría de la diuresis. Se logró extubación a las 12 horas post procedimiento. A los 6 días de evolución, se retiró sin inconvenientes el drenaje, sin reproducción posterior del quilotórax. Recibió dieta hipograsa por tres semanas en total. La paciente se dio de alta a los 46 días de hospitalización en buenas condiciones.

\section{Discusión}

El quilotórax a tensión es una entidad poco frecuente, con pocos casos reportados en la literatura, principalmente lo encontramos en población adulta, con etiología principalmente traumática y post quirúrgica (neumonectomia) ${ }^{8-14}$. Las formas de presentación fueron tan variadas con síntomas similares a taponamiento cardíaco, abdomen agudo ${ }^{10}$ o episodio sincopal $^{12}$.

Sólo en una reciente publicación Wheeler y Tobias ${ }^{15}$, comunican dos casos pediátricos, uno 
de ellos de cuatro semanas de edad, quién presentó quilotórax hipertensivo después de una cirugía de Fontan y el otro en un recién nacido de tres semanas de vida, quién cursó con un shock séptico, donde pudiese haber jugado un rol en el desarrollo del quilotórax el estado pro coagulante propio de la sepsis ${ }^{16}$, como también la presencia de un catéter central en las cercanías del ducto toráxico. Es importante recalcar que en los recién nacidos, intentos reiterados de ubicar una vía venoso central cerca del ducto toráxico por vía periférica, pueden ocasionar trauma local en la pared del vaso y eventualmente ocasionar quilotórax.

La presentación clínica en nuestros casos fue similar a la literatura revisada, correspondiendo a un grave compromiso hemodinámico, manifestado con taquicardia, mala perfusión periférica, hipotensión arterial y oliguria asociado a dificultad respiratoria progresiva, que ameritó necesidad de soporte ventilatorio artificial; por otra parte, se confirmó el rol trascendente del quilotórax, por la clara y rápida mejoría observada posterior al drenaje por toracocentesis de quilo a presión. También a similitud de uno de los casos previamente reportados ${ }^{15}$, los nuestros también ocurrieron luego de cirugía cardiotoráxica.

Se señala que el reconocimiento de esta entidad seria más fácil en aquellos pacientes que se presentan fuera del ambiente de cuidados intensivos, ya que en ellos los síntomas son los clásicos de la ocupación pleural, sin embargo, en ocasiones no resulta tan simple el diagnóstico de ocupación pleural a tensión cuando la causa no corresponde a aire, y que en general en algunos casos los síntomas pueden ir ocurriendo en forma progresiva, siendo el compromiso hemodinámico el evento final de esta secuencia 5 .

Por el contrario, en el contexto de un paciente post operado cardiotoráxico críticamente enfermo, quién no ha comenzado a alimentarse, se debe de tener un alto índice de sospecha ya que en estos casos las manifestaciones corresponderán a oliguria, aumento de los requerimientos de oxígeno, dificultad respiratoria, todos elementos inespecíficos que deben buscarse en forma dirigida ${ }^{15}$. Además el aspecto lechoso clásico del líquido puede variar, presen- tando una apariencia sero-sanguinolenta en etapa de ayuno, por lo tanto, un débito excesivo obtenido por cualquier drenaje pleural o el cambio de sus características macroscópicas al alimentarse, deben originar sospecha de un posible daño del ducto toráxico. Existen diversos factores que puede modificar la intensidad de los síntomas observados en los pacientes como son el volumen y la rapidez de la instalación del fluido acumulado, lo adecuado de la precarga en ese momento y la condición hemodinámica y ventilatoria previa.

Así entonces, en pacientes con factores de riesgo para el desarrollo de quilotórax (cirugía toráxica, trombosis venosa, shock séptico), se debe considerar también esta patología en el diagnóstico diferencial de la ocupación pleural con repercusión hemodinámica y ventilatoria.

Referente a la etiología del quilotórax, es conocido que esta puede ser de origen traumático, como la observada posterior a cirugía cardiotoráxica $^{17-19}$, o trauma toráxico penetrante o no traumáticas, como en hipertensión de la vena cava superior, trombosis venosa local. Finalmente, el quilotórax congénito puede ser visto en pacientes con anormalidades linfáticas (Síndrome de Down, Síndrome de Noonan, Síndrome de Turner), patologías linfáticas familiares como enfermedad de Milroy-Meige, entre otras $^{20}$.

Con respecto a las opciones de tratamiento del quilotórax, obviamente en situaciones en que este constituye una emergencia se requieren de una toracocentesis evacuadora e instalación de un tubo pleural. Cuando el quilotórax se pesquisa antes de estar a tensión se puede tratar con toracocentesis para drenar el quilo, modificación de la dieta con triglicéridos de cadena media como principal aporte lipídico o uso de nutrición parenteral total, limitando así la producción de quilo y asegurar una adecuada reposición de proteínas y nutrientes perdidos ${ }^{21}$. Otra opción terapéutica utilizada, es el uso de octreotido, análogo de la somastostatina, con resultados variables en su eficacia ${ }^{22}$.

Habitualmente la efusión de quilo cesa al final de la segunda semana de tratamiento, en los casos aquí comunicados el drenaje se pudo retirar a los 6 y 7 días respectivamente de instalado y no se utilizaron otros tratamientos 
más que el drenaje del quilo y modificación de la dieta.

Las indicaciones quirúrgicas son variadas, dependiendo de la patología de base, constituyéndose en la primera alternativa en casos de postneumonectomía o esofagectomia transhiatal (población adulta). En otros casos la indicación quirúrgica dependerá en general de la magnitud y duración del drenaje quiloso y sus consecuentes secuelas de pérdida de triglicéridos, proteínas, albúmina, inmunoglobulinas y descenso del recuento linfocitario. Las opciones quirúrgicas consisten en la ligadura del ducto toráxico, pleurodesis con talco, shunt pleuroperitoneal y cateterización percutánea con embolización del ducto toráxico.

\section{Conclusión}

Este reporte nos enseña que en determinados pacientes se debe considerar el diagnóstico de quilotórax a tensión como una entidad capaz de producir compromiso hemodinámico y respiratorio significativo y que su reconocimiento precoz permite revertir rápidamente esta situación potencialmente grave.

\section{Referencias}

1.- Efrati O, Barak A: Pleural effusions in the pediatric population. Pediatr Rev 2002; 23: 417-26.

2.- Leigh-Smith S, Harris T: Tension pneumothorax-time for a re-think? Emerg Med J 2005; 22: 8-16.

3.- Whiteman PJ, Wilson MT, Barcay D, Ting PP, Chen $S C$ : Tension pyopneumothorax in a child: a case report. J Emerg Med 2003; 24: 429-31.

4.- Samovsky M, Loberant N, Lemer J, Altman E: Tension pyopneumothorax. Clin Imaging 2005; 29: 437-8.

5.- Horst M, Sacher P, Molz G, Willi UV, Meuli M: Tension gastrothorax. J Pediatr Surg 2005; 40: 15004.

6.- Beach C, Manthey DE: Tension hydrothorax due to ventriculopleural shunting. J Emerg Med 1998; 16 : 33-6.

7.- Weber SM, Aihara R, Hirsch EF: Case of traumatic tension urohemothorax. J Trauma 2003; 54: 1253.

8.- Kanjanauthai S, Kanluen T, Bergman M: Tension Chylothorax: A Rare Life Threatening Entity After Pneumonectomy. Heart Lung Circ 2008; Jan 29 [Epub ahead of print].

9.- Ammori JB, Pickens A, Chang AC, Orringer MB: Tension chylothorax. Ann Thorac Surg 2006; 82: 729 30.

10.- Huwer H, Volkmer I, Feindt P: Tension chylothorax following left-sided lung surgery. Pneumologie 1991; 45: 337-9.

11.- Karwande SV, Wolcott MW, Gay WA Jr: Postpneumonectomy tension chylothorax. Ann Thorac Surg 1986; 42: 585-6.

12.- Jenkins JL, Dillard F, Shesser R: Tension chylothorax caused by occult trauma. Am J Emerg Med 2004; 22: 321-3.

13.- Glyn-Jones $S$, Flynn J: Traumatic tension chylothorax. Injury 2000; 31: 549-50.

14.- Chamberlain $M$, Ratnatunga $C$ : Late presentation of tension chylothorax following blunt chest trauma. Eur J Cardiothorac Surg 2000; 18: 357-9.

15.- Wheeler AD, Tobias JD: Tension chylothorax in two pediatric patients. Pediatric Anesthesia 2007; 17: 48891.

16.- Berkenbosh JW, Monteleone PM, Tobias JD: Chylotorax following apparently spontaneous central venous thrombosis in a patient with septic shock. Pediatr Pulmonol 2003; 35: 230-3.

17.- Chan EH, Russell JL, Williams WG, Van Arsdell GS, Coles JG, McCrindle BW: Postoperative chylothorax after cardiothoracic surgery in children: Ann Thorac Surg 2005; 80: 1864-70.

18.- Chan SY, Lau W, Wong WH, Cheng LC, Chau AK, Cheung YF: Chylothorax in children after congenital heart surgery. Ann Thorac Surg 2006; 82: 1650-6.

19.- Rodríguez J, Córdova G, Arretz C, et al: Experiencia clínica: Quilotórax en cirugía cardiovascular pediátrica. Rev Chil Pediatr 2003; 74: 53-9.

20.- Wegner A, Wegner ME, Milad M: Quilotórax en el período neonatal: caso clínico y revisión de la literatura. Rev Chil Pediatr 1999; 70; 6: 498-504.

21.- Büttiker V, Fanconi S, Burger R: Chylothorax in children: guidelines for diagnosis and management. Chest 1999; 116: 682-7.

22.- Epaud R, Dubern B, Larroquet M, et al: Therapeutic strategies for idiopathic chylothorax. J Ped Surg 2008; 43: 461-5. 